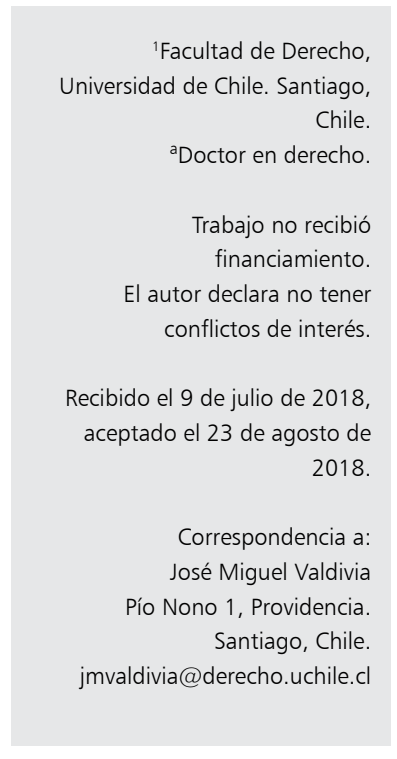

\section{La culpa médica en la responsabilidad de los hospitales públicos}

\author{
JOSÉ MIGUEL VALDIVIA ${ }^{1, a}$
}

\section{Medical malpractice in public hospitals}

Under Chilean public law, liability of a public hospital appears when the institution fails to accomplish its mandatory duties or incurs in negligence. As in private law liability, this system requires to determine the duties of hospitals and, in case of a medical accident, professional standards or duties. This paper explores the two main categories of medical malpractice, namely erroneous diagnosis and treatment failures based on public law theory and judiciary methods.

(Rev Med Chile 2018; 146: 1028-1032)

Key words: Hospital, Public; Liability, Legal; Malpractice.

\section{El sistema de la responsabilidad por culpa}

$\mathrm{P}$ ara los establecimientos públicos de salud el régimen de responsabilidad pecuniaria (i. e., indemnización de perjuicios) sigue las orientaciones generales del derecho público: corresponde a la responsabilidad por falta de servicio, un tipo de responsabilidad por culpa. Esta responsabilidad rige para la generalidad de los servicios públicos, incluidos los servicios de salud, desde 1986 y, más explícitamente, a partir de 2004, respecto de todos "los órganos de la Administración del Estado en materia sanitaria"1.

La noción central del régimen es la de falta de servicio, traducción casi literal de la noción francesa de faute de service. La falta de servicio corresponde, en general, a una culpa institucional de los organismos públicos ${ }^{2}$. Atendida la generalidad de su ámbito de aplicación, no es extraño que la falta de servicio se conciba en términos abstractos, como incumplimiento de un patrón de conducta esperable del servicio público. Por cierto, su concretización depende de los deberes o estándares de servicio exigibles de cada tipo de institución pública. Por eso, en la medicina pública, supone definir los deberes del establecimiento sanitario para con sus usua- rios $^{\mathrm{i}}$, sea que estos deberes conciernan al aparato administrativo o al desempeño de su personal, incluidos los profesionales de la salud.

Este régimen supedita la indemnización a la demostración de una falla o anomalía en el funcionamiento del establecimiento sanitario. En la práctica el sistema opera de modo similar a la responsabilidad civil de hospitales y clínicas privadas, articulada en torno a la noción de culpa. La similitud alcanza a las nociones de falta de servicio y culpa, construidas sobre la base de deberes que, en cuanto derivan de una misma actividad, son relativamente homogéneos.

Lógicamente, este sistema se aparta de la responsabilidad objetiva. Una responsabilidad es objetiva (o sin culpa) cuando para que se vea comprometida basta con la ocurrencia de un daño provocado por un hecho ocurrido dentro del ámbito de riesgos del responsable, aunque ese hecho no se pueda calificar como culpable. En el campo médico, aceptar un sistema así presentaría el riesgo de que el Estado tuviera que hacerse cargo de todas las consecuencias del estado de salud del paciente, desde que éste se pone bajo el cuidado del servicio

i Con todo, también hay falta de servicio en la infracción de deberes del hospital para con terceros o sus funcionarios: Corte Suprema, 14 de agosto de 2017, Rol 95.182-2016. 
público. Semejante régimen supondría un modelo muy costoso de seguridad social, que requeriría un acuerdo legislativo importante.

\section{La noción de culpa en la falta de servicio de los hospitales}

Entendida como incumplimiento de deberes o estándares de actuación, en el campo médico la falta de servicio no puede darse por establecida únicamente a partir de un resultado perjudicial para el paciente. A menudo el estado de salud del paciente trae el germen del daño, de modo que la obligación del establecimiento hospitalario no puede consistir en curarlo, sino en tratarlo adecuadamente. En términos análogos al derecho civil contractual, el sistema de responsabilidad no puede reposar en un concepto mecánico de culpa, como aquel a que conduciría una obligación de resultado, en que el resultado sería la mejoría del paciente. Al contrario, el establecimiento de la culpa exige demostrar la transgresión de un estándar de diligencia, que es consistente con los deberes prudenciales asociados al tipo de decisión que deben tomar los profesionales de la saludii. Por cierto, nada impide que algunas hipótesis de falta de servicio puedan establecerse a partir del incumplimiento de deberes suficientemente precisos.

El sistema legal recoge indirectamente esta concepción a propósito del régimen probatorio. $\mathrm{La}$ ley dispone que el demandante "deberá acreditar que el daño se produjo por la acción u omisión del órgano, mediando dicha falta de servicio". Literalmente, la regla consagra un régimen de culpa probada. Sin embargo, al dispositivo probatorio subyace un modelo de distribución de riesgos que apunta en esta dirección: la víctima no será indemnizada a partir de las simples consecuencias de su afección inicial o de su evolución normal o previsible, sino únicamente si logra demostrar que el daño sobrevino o se agravó por una causa distinta, imputable al servicio y susceptible de calificarse como falta de servicio.

La determinación concreta de la falta de servicio depende de los deberes contextuales del establecimiento sanitario en las circunstancias del daño. En otros términos, para estimar que el hos-

ii Corte Suprema, 30 de noviembre de 2009, Rol 6384-2008. Descartando la idea de una obligación de resultado. pital ha incurrido en falta de servicio es necesario atender al tipo de situación en que acaece el daño; si éste ocurre en razón del desempeño del médico, la determinación de la falta de servicio exigirá apreciar los deberes propios del profesional.

\section{La culpa individual del médico en la responsabilidad por falta de servicio}

La falta de servicio supone una especie de culpa atribuible al servicio público y, consistentemente, la indemnización grava el patrimonio del hospital o del Fisco. Sin embargo, la falta de servicio siempre tiene por origen hechos imputables al personal de los servicios públicos; "el servicio" es una alegoría jurídica, pues los únicos que pueden incurrir en culpa son las personas de carne y hueso que lo animan. Por eso, cuando el demandante alega que el daño tiene por origen la mala atención de un médico, es ineludible revisar su intervención personal en los hechos a la luz de sus deberes (ante todo, profesionales).

El factor personal está en el corazón mismo del mecanismo de responsabilidad. El organismo estatal responde si incurre en falta de servicio pero, además, puede recuperar las indemnizaciones que hubiere pagado en razón de una "falta personal" o, tratándose de profesionales de la salud, "imprudencia temeraria o dolo". El dispositivo legal revela que el patrimonio estatal absorbe tanto las indemnizaciones que se originen en una falta de servicio como aquellas que surjan de una falta personal, que es una culpa singularizada del médico, revestida de cierta gravedad. Con todo, es un dato bien conocido que la falta personal rara vez es perseguida por la administración, tanto en general, como específicamente en el ámbito médico (lo cual puede explicarse por el alto estándar fijado por la ley, propio de conductas delictivas).

Ahora bien, en la falta de servicio ese factor individual suele diluirse, en razón de la multiplicidad de intervinientes en la gestión de un servicio público. Las instituciones administrativas son organismos complejos, compuestos por varios agentes, medios materiales apropiados y una administración que gestiona esos distintos recursos y sus necesidades. Por eso, desde su teorización temprana por la doctrina francesa, la falta de servicio ha presentado un cierto carácter colectivo o 
anónimo en que la individualidad de los agentes públicos se funde en la gestión pública ${ }^{4}$.

También es así en el terreno hospitalario. La jurisprudencia abunda en referencias a faltas de servicio cometidas por el "equipo médico", sin especificar sus integrantes ni la intervención que hubieren tenido. Por lo demás, aun sin radicar la culpa en entelequias colectivas, bajo la idea de una mala atención brindada al paciente muchas veces se esconden comportamientos atribuibles a distintas categorías de profesionales o simples agentes administrativos. Este proceder ciertamente dificulta la identificación de la culpa médica.

\section{La especificidad de la culpa médica}

A pesar de su dificultad, puede ser útil deslindar el campo preciso de intervención del médico respecto de los demás agentes que participan en el hospital. En cuanto la falta de servicio supone infracción de deberes de servicio, la determinación de estos deberes ( $y$, correlativamente, de las expectativas del público) varía en función del tipo de actividad hospitalaria de que se trate. Luego, este esfuerzo de distinción contribuye al mejor funcionamiento del régimen de responsabilidad y al propósito pedagógico u orientativo de todo sistema basado en la culpa.

En principio, la actividad médica está sujeta a deberes que derivan de la complejidad de la profesión y de las circunstancias en que se ejerce, en la que intervienen frecuentemente juicios técnicos y prudenciales que no pueden reducirse a un catálogo predefinido. En contraste, los deberes exigibles en el desempeño de las actividades no médicas (por personal paramédico y administrativo), presentan una relativa simplicidad, que facilita su verificación ex post por los jueces.

Esta distinción aparece esbozada en el sencillo caso Morales Quezada, relativo a un accidente ocurrido durante el transporte de un paciente en camilla, en que la justicia descartó la defensa del hospital consistente en que no se había violado la lex artis. La Corte tuvo ocasión de afirmar que el examen de las reglas del arte sólo es pertinente para juzgar la responsabilidad en que se incurre por actividades médicas propiamente tales y no aquellas que pueden confiarse al personal menos calificado ${ }^{\text {iii. }}$.

iii Corte Suprema, 6 de noviembre de 2012, Rol 5417-2012.
Los casos más frecuentes en que se reconoce la culpa médica corresponden a defectos de diagnóstico y de materialización de un tratamiento.

\section{Defectos de diagnóstico}

La jurisprudencia llama la atención sobre los errores incurridos en el diagnóstico de la dolencia del paciente. Este enfoque tiene sentido (por así decir, procedimental) porque el diagnóstico es la primera fase de la intervención del médico: al menos cronológicamente se sitúa en el origen de un tratamiento potencialmente inapropiado ${ }^{\text {iv }}$. Se advierten dos métodos de establecimiento del error de diagnóstico.

De modo ortodoxo, la jurisprudencia reconoce un error de diagnóstico cuando el curso de acción dispuesto por el médico no aparece respaldado por los antecedentes técnicos con que cuenta. Esto ocurre típicamente cuando un médico se aparta de los resultados de los exámenes relativos al paciente. Así, en Salgado Durán la paciente es sometida a exámenes que sugieren una trombosis, los que después no son tomados en cuenta, tanto para disponer el alta como para su atención posterior ${ }^{\mathrm{v}}$.

En contraste, la jurisprudencia recurre a un método cuestionable de establecimiento del error de diagnóstico a partir de la simple circunstancia de que el médico no haya detectado la dolencia que aqueja al paciente. En Pizarro Araya, por ejemplo, la Corte Suprema declara la falta de servicio porque no consta "que durante las consultas médicas se le hubieran efectuado las pruebas y exámenes necesarios para descartar" determinado síndrome ${ }^{\text {vi }}$. Este método es problemático pues defrauda el principio de la culpa probada. Asumiendo que siempre se puede precisar ex post la dolencia específica de un paciente, el médico incurriría sistemáticamente en culpa cada vez que no logre averiguarla, con prescindencia de sus esfuerzos, de los medios técnicos disponibles, de la complejidad del caso y de su urgencia. En otros términos, de la causa

iv Así, Corte Suprema, 24 de octubre de 2016, Rol 28.5862016 (bronconeumonía tratada como bronquiolitis).

v Corte Suprema, 31 de julio de 2017, Rol 8355-2017.

vi Corte Suprema, 16 de mayo de 2016, Rol 18124-2015 (síndrome compartimental). 
inmediata del daño, proveniente de la patología o condición de salud del paciente, se inferiría la culpa del médico.

En verdad, esta orientación jurisprudencial desafía aquella que revela que en otras circunstancias es el mismo estado de salud del paciente lo que dificulta efectuar diagnósticos certeros ${ }^{\text {vii. }}$.

Es posible que en algunos casos la sintomatología del paciente no deje espacio a dudas respecto del mal que lo aqueja, de modo que hay falta de servicio al no saber apreciarlo sin necesidad de

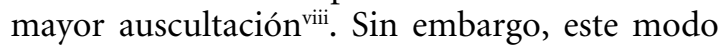
de formular un reproche sólo puede aceptarse en caso del diagnóstico fallido de afecciones sencillas o evidentes.

En fin, la jurisprudencia también da cuenta de otras faltas de servicio cometidas con relación al diagnóstico pero que no envuelven necesariamente errores de juicio; por ejemplo, si el diagnóstico no es comunicado al paciente ${ }^{\mathrm{ix}} \mathrm{o}$ se producen demoras en el procedimiento ${ }^{\mathrm{x}}$. Sin embargo, muchas de ellas son provocadas por la acción (exclusiva o concurrente) del personal administrativo del hospital.

\section{Defectos de materialización del tratamiento}

Un segundo grupo de casos en que la culpa del médico es reconocida con relativa sencillez corresponde a errores o inconsistencias en la administración del tratamiento.

Es difícil de olvidar el caso Beraud, en que estando programada una operación de cadera derecha, el equipo médico intervino la izquierda ${ }^{x i}$. Años más tarde se reconoce la falta de servicio en el caso de un paciente operado sobre la base del diagnóstico correspondiente a otra persona $\mathrm{a}^{\mathrm{xii}}, \mathrm{o}$ sometido a un procedimiento distinto del pro-

vii Corte de Valparaíso, 2 de septiembre de 1999, Rol 29341998 (apendicitis) o Corte Suprema, 15 de noviembre de 2012, Rol 2411-2012 (carcinoma mamario).

viii Parece ser el caso de Corte Suprema, 4 de julio de 2017, Rol 95.114-2016 (shock anafiláctico experimentado en el establecimiento sanitario).

ix Corte Suprema, 19 de mayo de 2015, Rol 4388-2015.

x Corte Suprema, 14 de septiembre de 2016, Rol 35.5662015.

xi Corte Suprema, 20 de junio de 1996, Rol 33.393-1995.

xii Corte Suprema, 1 de junio de 2017, Rol 87.914-2016. gramado $^{\text {xiii }}$. Igualmente, hay falta de servicio si se olvida una intervención que debía practicarse ${ }^{\mathrm{xiv}}$, o no se cumple la orden de trasladar el paciente a una unidad especializada ${ }^{\mathrm{xv}}$. El retraso en la práctica de una actuación relevante también puede calificarse como falta de servicio, como ocurre típicamente en la atención de partos ${ }^{\mathrm{xvi}}$. Aquí también, la falta de servicio no siempre es exclusivamente imputable al médico sino a otros agentes del hospital.

En el ámbito quirúrgico, y no obstante su complejidad de principio, la falta de servicio puede establecerse a partir de la impericia del médico, como en caso de lesiones o heridas causadas durante una operación, pero sin relación funcional con la intervención misma ${ }^{\text {xvii }}$. También puede establecerse a partir del incumplimiento de deberes de orden práctico que no impliquen la toma de decisiones delicadas, como ocurre en el olvido de compresas o instrumental dentro del cuerpo del paciente ${ }^{\mathrm{xviii}}$.

Por la importancia creciente de los derechos del paciente, cabe también llamar la atención sobre la inobservancia de las exigencias de recabar el consentimiento informado frente a algún tratamiento ${ }^{\mathrm{xix}}$.

\section{Métodos de reconocimiento de la falta de servicio médica}

Para la jurisprudencia, la culpa médica aparece preferentemente como un apartamiento de las orientaciones referenciales contenidas en protocolos o guías clínicas. Teóricamente, la culpa médica supone infracción a la lex artis, pero ésta no es una auténtica ley sino un conjunto más o menos inorgánico de criterios valorativos propios

xiii Corte Suprema, 19 de mayo de 2016, 32.268-2015 (vaciamiento axilar en vez de biopsia).

xiv Corte Suprema, 29 de enero de 2016, Rol 13.544-2015 (esterilización no practicada; es un caso de wrongful pregnancy).

xv Corte Suprema, 6 de junio de 2016, Rol 1574-2016.

xvi Por ejemplo, Corte Suprema, 2 de agosto de 2016, Rol 16.002-2016.

xvii Corte Suprema, 28 de junio de 2016, Rol 34.161-2015.

xviii Corte Suprema, 31 de julio de 2012, Rol 9140-2009.

xix Corte Suprema, 3 de septiembre de 2015, Rol 9058-2015 (esterilización no consentida). 
del oficio. Eventualmente los protocolos pueden facilitar el reconocimiento de las reglas del arte (es más, un fallo Morales Cancino habla de "protocolo constitutivo de la lex artis" ${ }^{\mathrm{xx}}$ ). Por esta vía, un comportamiento distinto al sugerido por el protocolo médico puede tenerse por infracción a la lex artis. El reconocimiento de la culpa médica se ve facilitado por esta "codificación" flexible de los deberes de conducta asociados a determinados tratamientos. Este método ofrece dos ventajas a la jurisprudencia. Por una parte, simplifica el establecimiento de deberes inherentes a una disciplina científica en la que los jueces normalmente no tienen expertise; en vez de arriesgarse a descubrir esos deberes, los jueces descansan en la autoridad de los protocolos (independientemente de su déficit normativo o de su carácter no exhaustivo). Por otra parte, la falta de servicio aparece constituida, como si fuese una culpa infraccional, por el solo hecho de transgredirse alguna de las recomendaciones contenidas en estos documentos, de un modo relativamente mecánico.

Paradójicamente, la frecuencia con que son reconocidas faltas de servicio de diagnóstico y de ejecución del tratamiento sugiere que la prescripción del tratamiento es difícilmente censurable ${ }^{\mathrm{xxi}}$. El peso estadístico del error de diagnóstico revela que la jurisprudencia prefiere detectar fallas en el procedimiento que condiciona el tratamiento, antes que juzgar su mérito intrínseco. Sin embargo, este método "procedimentalista" genera la apariencia engañosa de que el diagnóstico es una operación mecánica y no un ejercicio dominado también por criterios prudenciales. Por su parte, la recurrencia de las faltas cometidas en la administración material del tratamiento sugiere que, para los jueces, una vez decidido el tratamiento sólo cabe ejecutarlo con fidelidad. Aunque aquí

xx Corte Suprema, 8 de agosto de 2017, Rol 97.628-2016.

xxi Corte Suprema, 6 de julio de 2018, Rol 38.838-2017 (prescripción de medicamento convulsivo, inapropiado para paciente epiléptico). también caben juicios prudenciales, en la práctica parece más sencillo controlar estos aspectos que penetrar en la justificación técnica del tratamiento.

\section{Conclusiones}

La adopción del estándar de la falta de servicio en el campo médico presenta la ventaja de la coherencia con el régimen común de responsabilidad civil, dada su estrecha semejanza con la noción civil de culpa. Siguiendo orientaciones históricas, era de esperar que en el establecimiento de la falta de servicio los jueces mostrasen deferencia para con los cultores de una disciplina científica de alta complejidad como la medicina. Este marco analítico no ha perdido pertinencia, aunque las técnicas jurisprudenciales empleadas en este terreno estén trivializando el reconocimiento de la falta de servicio. Estos criterios permiten a la jurisprudencia una evolución matizada desde una mayor deferencia por la medicina hacia una mayor sensibilidad por los usuarios del servicio público.

\section{Referencias}

1. Artículo 42 de la Ley 18.575 (1986). Ley orgánica constitucional de bases generales de la Administración del Estado. Diario Oficial de la República de Chile. Link: http://bcn.cl/1uw4b. Artículos 38 y ss. de la Ley 19.966 (2004). Ley que establece un régimen de garantías en salud. Diario Oficial de la República de Chile. Link: http://bcn.cl/1uyp6.

2. Barros E. Tratado de responsabilidad extracontractual. Santiago: Editorial Jurídica, 2006; pp. 485 y ss.

3. Artículo 38, inciso 3 de la Ley 19.966 (2004). Ley que establece un régimen de garantías en salud. Diario Oficial de la República de Chile. Link: http://bcn.cl/1uyp6.

4. Paul Duez V. La responsabilité de la puissance publique (en dehors du contrat). París: Dalloz, 2a ed., 1938; pp. 21 y ss. 